\title{
The exoplanet next door
}

\section{Earth-sized world discovered in nearby $\alpha$ Centauri star system.}

\section{BY ERIC HAND}

$\mathrm{I}$ $\mathrm{t}$ is a world so close that E.T. could phone home in just four years. Astronomers have discovered the lowest-mass planet yet orbiting a Sun-like star. It orbits a Centauri B, a member of the stellar system that is our Solar System's nearest neighbour. Although nearly identical to Earth in mass, the planet is much closer to its star than Mercury is to the Sun, meaning that it is a scorched and barren rock. Nevertheless, its astronomical proximity to Earth will undoubtedly stir dreams of interstellar exploration, particularly as astronomers search a Centauri for more hospitable worlds.

Such exoplanets are the closest astronomers can ever find, says Francesco Pepe, an astronomer at the University of Geneva, Switzerland, and a member of the team that reports the discovery online in Nature this week (X. Dumusque et al. Nature http://dx.doi. org/10.1038/nature11572; 2012).

The work is a triumph for a venerable planet-finding technique that monitors a star for a subtle back-and-forth 'wobble' in its motion as seen from Earth, caused by the gravitational tug of an orbiting body. The technique, which formally measures a star's radial velocity, enabled researchers at Geneva to find in 1995 the first exoplanet ever detected orbiting a Sun-like star (M. Mayor and D. Queloz Nature 378, 355-359; 1995).

That planet, a gas giant called 51 Pegasi b, pulled its star at 50 metres per second. The motion of a Centauri B arising from its lightweight planet is 100 times slower, a mere 50 centimetres per second, akin to a slow walk. That the team detected it at all is testimony to the steady refinement of the technique (see 'Lowest of the low').

Pepe and his colleagues found the planet as part of a campaign to monitor ten of the brightest and nearest stars visible from the Southern Hemisphere using the High Accuracy Radial Velocity Planet Searcher (HARPS) spectrograph on the European Southern Observatory's 3.6-metre telescope at La Silla in Chile. Three of the stars have already yielded planets, and the a Centauri system became the fourth after the team measured its velocity three times a night, nearly every night, for just over three years, to tease out the subtle motion measured just above the detection limit.

Finding a true Earth twin, circling a Sun-like

\section{DNATURE.COM} For a News \& Views discussing this discovery see: go.nature.com/bxtcii

\section{LOWEST OF THE LOW}

With improving techniques for measuring exoplanets' mass through their gravitational influence on stars, the lightest exoplanets detected each year (red dots) have reached the range of Earth-mass planets.

100

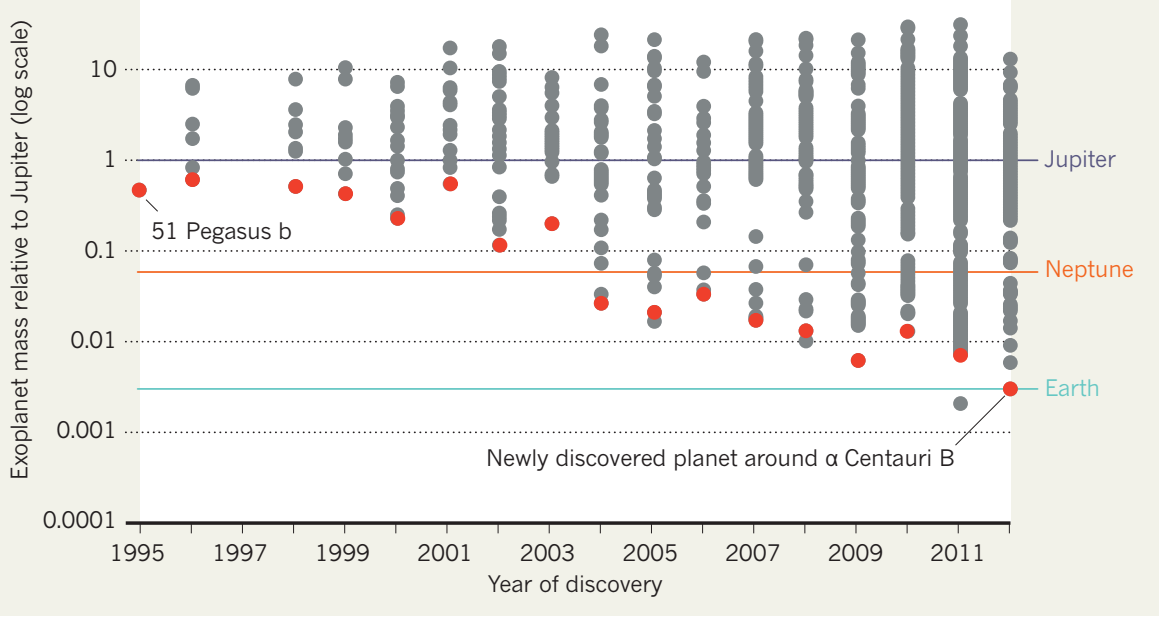

star at a distance similar to Earth's orbital radius around the Sun, would require another leap in sensitivity, to motions of just 9 centimetres per second. For now, most astronomers are pinning their hopes of identifying an exo-Earth on a rival planet-finder: the NASA spacecraft Kepler, which watches for a dimming in a star's light as a planet crosses its face. Kepler was launched in 2009, and data from its first two years of operation have revealed about 3,000 candidate exoplanets, says principal investigator William Borucki, who presented fresh results on 15 October at a meeting of the Division of Planetary Sciences of the American Astronomical Society in Reno, Nevada.

Surprisingly, the cumulative data set does not contain a single Earth-sized planet at a habitable distance from its star, according to Borucki, who is based at NASA Ames Research Center in Moffett Field, California. Earth-sized planets may simply be less common than larger ones, perhaps because they are less apt to form, he said, flashing up a slide suggesting that just $10-15 \%$ of stars have Earth-sized planets - far fewer than other estimates.

Glumly, Borucki also pointed out that Kepler is now working on borrowed time. One of the spacecraft's four reaction wheels, used to keep the probe pointing at its target, failed in July, and if one of the other wheels follows suit, the mission would be doomed.

Yet an Earth twin could in principle be hiding at a Centauri, which is actually a trio of stars some 1.34 parsecs (4.4 light years) away. Two of the stars in the system, A and $\mathrm{B}$, sometimes pass as near to each other as Saturn is from the Sun. This could disrupt the orbits of planets much farther out than the one discovered by the HARPS team, but a planet orbiting in a Centauri B's habitable zone, about half as far from the star as the distance between Earth and the Sun, would probably still be stable.

Geoff Marcy, an astronomer at the University of California, Berkeley, who leads another ground-based search effort, says that the discovery should bolster calls for a space-based telescope that could image any other planets near a Centauri B, providing they are in orbits large enough to separate them from the glare of the parent star. Such a telescope could also glean clues about a planet's atmospheric composition from its spectrum.

A space-based planet imager is still largely a dream, let alone an interstellar probe that could inspect the a Centauri system close up. Ralph McNutt, a planetary scientist at Johns Hopkins University Applied Physics Laboratory in Laurel, Maryland, has received NASA study funding to design an 'innovative interstellar explorer'. Even when launched by one of the most powerful rockets on Earth, boosted by a gravitational slingshot around Jupiter, and further accelerated by a radioisotope thruster, that probe would take about 28,000 years to reach a Centauri. Quoting British author Douglas Adams, McNutt quips: "Space is big. Really big." - 